\title{
Is Visual Imagery Really Visual? Overlooked Evidence From Neuropsychology
}

\author{
Martha J. Farah \\ Carnegie-Mellon University
}

\begin{abstract}
Does visual imagery engage some of the same representations used in visual perception? The evidence collected by cognitive psychologists in support of this claim has been challenged by three types of alternative explanation: Tacit knowledge, according to which subjects use nonvisual representations to simulate the use of visual representations during imagery tasks, guided by their tacit knowledge of their visual systems; experimenter expectancy, according to which the data implicating shared representations for imagery and perception is an artifact of experimenter expectancies; and nonvisual spatial representation, according to which imagery representations are partially similar to visual representations in the way they code spatial relations but are not visual representations. This article reviews previously overlooked neuropsychological evidence on the relation between imagery and perception, and discusses its relative immunity to the foregoing alternative explanations. This evidence includes electrophysiological and cerebral blood flow studies localizing brain activity during imagery to cortical visual areas, and parallels between the selective effects of brain damage on visual perception and imagery. Because these findings cannot be accounted for in the same way as traditional cognitive data using the alternative explanations listed earlier, they can play a decisive role in answering the title question.
\end{abstract}

The question of whether visual imagery is really visual, that is, whether it involves some of the same representations of stimuli normally engaged by the perception of those stimuli, has been the subject of a long-standing debate in cognitive psychology. This article reviews a set of empirical findings from neuropsychology that are directly relevant to this debate. I will argue that this generally overlooked source of data can play an important role in determining the relation between imagery and perception, because it is immune to many of the criticisms and alternative explanations that have plagued the cognitive psychology approach to this topic.

One side of the debate maintains that imaging consists of the top-down activation of perceptual representations, that is, representations that are also activated automatically by an external stimulus during perception. This idea dates back at least as far as the philosophical writings of Hume (1739/1969) and has been put forth more recently by Hebb (1968), Shepard (1978, 1984) and Finke (1980). In contrast, the other side of the debate maintains that the representations used in imagery are not the

The writing of this article was supported by Office of Naval Research Contract N00014-86-K-0094, National Institutes of Health (NIH) Grant NS23458, the Alfred P. Sloan Foundation Program in Computational Neuroscience, and NIH Program Project Grant NS 06209-21 to the Aphasia Research Center of the Boston University School of Medicine.

The author thanks Jennifer Brunn, Michael Corballis, Ron Finke, Clark Glymour, Margaret Intons-Peterson, Ross Thompson, and two anonymous reviewers for their helpful comments on an earlier draft of this article.

Correspondence concerning this article should be addressed to Martha J. Farah, Department of Psychology, Carnegie-Mellon University, Pittsburgh, Pennsylvania 15213-3890. representations used in perception, and that the recall of visual information, even when accompanied by the phenomenology of "seeing with the mind's eye," is carried out using representations that are distinct from those used in veridical seeing (Pylyshyn, 1973, 1978, 1984, chap. 8).

What is at stake in this debate, that it should continue to be a focus of research and discussion on mental imagery? To begin with, it is a basic question about the representations underlying mental imagery, and on these grounds alone it warrants focused attention from cognitive psychologists. In an early and influential critique of imagery research, Pylyshyn (1973) concurred with imagery researchers that this issue is central: "Atwood (1971) is quite right when he states, 'The most elementary question that can be asked about mnemonic visualization is the following: does the mnemonic image actually involve the visual system?" " A decade and a half of active research on this issue has ensued (see Finke, 1985, for a recent review). The resolution of this controversy would also have broader implications beyond our understanding of mental imagery per se: For example, if visual imagery does engage visual perceptual representations, then at least some perceptual representations are not "informationally encapsulated" (see Fodor, 1983) insofar as they may take input from higher cognitive processes (i.e., imagery) as well as from bottom-up perceptual processes triggered by external stimuli. Furthermore, such a conclusion would imply that thinking in images involves representations (in the perceptual system) that are distinct from the representations used in nonimagistic thought, in turn implying a modular structure for the representations underlying thought (cf. Anderson, 1983; Fodor, 1983).

In discussing the theoretical implications of the relation between imagery and perception, it is worth noting explicitly an issue for which this relation has no direct implications, namely, 
the issue of the format of mental images. Claims that images are pictorial or descriptive, array-like or propositional, analog or symbolic, are all claims about the format of images. As Block (1983) has pointed out, the relation of imagery to perception and the format of mental imagery are issues which are often conflated but are in principle independent. The finding that imagery shares representations with perception would not imply that imagery is pictorial; both imagery and perception might be descriptive. Furthermore, perceptual representations and mental images could have the same format (pictorial or descriptive) and yet be distinct representations.

\section{Previous Research in Cognitive Psychology}

Within cognitive psychology, several research programs have gathered evidence of common representations for imagery and perception. A comprehensive review of this work may be found in Finke (1985). A few representative examples of this large literature will be presented here.

Shepard's finding that shapes can be mentally reoriented only with a continuous "mental rotation" provided an early demonstration of the apparently visuospatial properties of mental images (see, e.g., Shepard \& Cooper, 1982). More recently, Shepard has explicitly related image rotation and other image transformations to the same mechanisms that underlie the visual perception of motion through comparative studies of image transformation and apparent motion (see, e.g., Shepard, 1984). In a separate series of studies with Podgorny (Podgorny \& Shepard, 1978), he has demonstrated the functional equivalence of mental images and visual percepts in a dot localization task: Subjects viewed a square grid in which they either imagined or were presented with a block letter. On each trial a probe dot was presented somewhere in the grid and the subjects' task was to decide whether the dot fell on or off the (real or imagined) letter. Podgorny and Shepard found that the pattern of response times were highly dependent on the spatial position of the dot with respect to the letter. More important, the pattern of response times was essentially the same whether the letter was real or imagined, as would be expected if images and percepts of the letters activated common representations.

Kosslyn's (1980) studies of mental imagery have been primarily aimed at elucidating the format of mental images and other information-processing characteristics of mental imagery, but some of his findings nevertheless bear on the relation between imagery and perception. For example, the finding that images have a limited resolution, such that two imaged points can only be brought so close before they fuse (Finke \& Kosslyn, 1980 ) and the finding that images show the visual "oblique effect," such that lines can be imaged more closely spaced at a horizontal or vertical orientation than obliquely (Kosslyn, 1983 , pp. 81-83) have been taken to imply that visual representations are being used.

Finke (e.g., 1980) has addressed the issue of the relation of imagery to perception directly in a series of striking experimental demonstrations of image-percept equivalence. He has found that mental images can function equivalently to visual percepts in producing visual-motor adaptation (Finke, 1979) and an orientation-specific color adaptation known as the McCollough effect (Finke \& Schmidt, 1977, 1978). Furthermore, he has shown that the functions describing the relation between resolution and eccentricity in the (real or imagined) visual field are highly similar for images and percepts (Finke \& Kosslyn, 1980; Finke \& Kurtzman, 1981). Each of these demonstrations of image-percept equivalence is consistent with the claim that some of the same representations are being used in imagery and perception.

\section{Alternative Explanations of Findings in Cognitive Psychology}

The foregoing evidence has not been viewed as decisive by all psychologists. Three different arguments have been put forth questioning the inferences that link the type of data collected by Shepard, Kosslyn, and Finke to the conclusion that imagery shares representations with perception.

Pylyshyn (1981) has argued that subjects in imagery experiments may use general-purpose cognitive processes (as opposed to specifically visual processes), along with tacit (i.e., unconscious) knowledge of how their visual systems behave, to simulate the use of their visual systems. Although the "tacit knowledge" account of imagery experiments was formulated by Pylyshyn initially as an alternative to claims about the pictorial format of images, it is equally powerful as an alternative to claims of shared representations for imagery and perception. For example, according to a tacit knowledge account, the amount of time it takes subjects to rotate an image from one orientation to another is linearly related to the angular difference between the two orientations, not, as Shepard (1984) has claimed, because visual mechanisms that evolved to perceive continuous rotational motion are being used. Rather, subjects tacitly know that when they actually see an object changing its orientation, it does so continuously and in an amount of time proportional to the angle through which it rotates. Believing their task to be one of simulating a visual experience, subjects therefore modulate their response times to conform with this tacit knowledge about perceived rotations. All of the evidence cited earlier in support of image-percept equivalence can in principle be accounted for in a similar way, by assuming that subjects have tacit knowledge of such properties of their visual system as its fields of resolution, the oblique effect, and various adaptation effects. In principle, without some independent way of verifying what subjects do and do not tacitly know about their own visual systems, researchers cannot exclude this type of alternative explanation of the large body of data in cognitive psychology showing visual properties of mental images.

The difficulty of replicating many of the more impressive findings of image-percept equivalence (Broerse \& Crassini, $1980,1981,1984$; Intons-Peterson \& White, 1981) has led some psychologists to consider the role of experimenter expectancy effects in imagery research. For example, Intons-Peterson (1983) has contended that the experimental paradigms used to study the visual properties of imagery are sufficiently vulnerable to experimenter expectancy that much, if not all, of the data showing visual properties of images could be artifactual. In support of this claim, she manipulated the expectations of research assistants regarding the outcome of a series of experiments on the relation of imagery to perception, and found that this systematically affected the results of the experiments. Note that 
the experimenter expectancy effects created by Intons-Peterson consisted of simple increases or decreases of imagery performance relative to perceptual performance, and not the precise quantitative similarities and interactions between imagery and perception that Finke, Shepard, and Kosslyn have found. However, the published failures to replicate some of these findings, along with the fact that positive findings of image-percept equivalence have invariably been obtained by researchers who believe that imagery shares representations with perception, gives plausibility to Intons-Peterson's claim and makes it, at present, impossible to reject.

A third challenge to the claim that visual imagery involves visual representations comes from research with congenitally blind subjects (e.g., Carpenter \& Eisenberg, 1978; Kerr, 1983; Marmor \& Zabeck, 1976; Zimler \& Keenan, 1983). In these studies, subjects who were blind from birth have been found to perform virtually normally on tasks such as image rotation, inspection, and scanning (in some of which tactile stimuli were substituted for the usual visual stimuli). For example, one of the tasks that Kerr (1983) adapted from the imagery literature was based on Kosslyn's (1975) image inspection task, in which he manipulated the size at which people imaged a given object by having them image it to scale next to an elephant (in which the case the image was small) or a fly (in which case the image was large). Kosslyn found apparent visual resolution effects: Subjects took longer to "see" the parts of objects in small images than in large images. Kerr instructed her congenitally blind subjects to image a familiar household object either next to a car or next to a paper clip, and then measured how long it took them to search their image for a particular named part, such as the dial on a radio. Just as Kosslyn had found with sighted subjects, Kerr found slower response times to find the named parts when the images were small. Her conclusion was that the representations used in imagery do indeed have spatial properties, like visual representations, but they need not be visual themselves; in fact, with the congenitally blind subjects they were certainly not visual. These and similar findings with congenitally blind subjects pose the following general problem for interpreting the results of the larger imagery literature with sighted subjects: If the reaction time effects observed by Kosslyn, Shepard, Finke, and others in visual imagery tasks can be observed in similar tasks with subjects who, because they have never seen, could not possibly be using visual information, then it is possible that the findings in sighted subjects are also due to the use of nonvisual spatial representations (cf. Baddeley \& Lieberman, 1980; Neisser \& Kerr, 1973).

The criticisms and alternative explanations of imagery research cited earlier are either in practice difficult or in principle impossible to reject using the conventional methods of cognitive psychology. For example, no matter how subtle and unexpected a perceptual property (like an acuity function) can be shown to manifest itself in imagery, psychologists cannot, in principle, know that subjects are not using tacit knowledge about this property of their visual systems. Tacit knowledge accounts may be more or less plausible, but for any conceivable finding of similarity between imagery and perception in a cognitive psychology experiment they will always be possible. Experimenter expectancy effects do not hold the same in-principle invulnerability to all possible data, but at present they are im- possible to reject, as virtually all of the published experiments on image-percept equivalence (along with most other experiments in cognitive psychology, of course) could conceivably have permitted the transmission of experimenter expectancies to the subjects. Finally, the research on imagery in the congenitally blind calls our attention to the existence of nonvisual representations with spatial properties similar to visual representations, which could in principle account for all of the findings so far attributed to the "visual" properties of imagery. ${ }^{1}$

We are therefore in a theoretical stalemate over the issue of the relation between imagery and perception: All of the experimental results in cognitive psychology that were initially taken to support the existence of shared representations for imagery and perception are now seen to be open to each of three different types of alternative explanation. One approach to breaking this stalemate would be to carry out another body of experiments similar to the ones just described but which use naive experimenters (to rule out experimenter expectancy effects), which involve properties of the visual system not shared with nonvisual representational systems (e.g., color, binocular effects, to rule out the use of amodal spatial representations) and which involve properties so subtle that tacit knowledge accounts become unacceptably strained. A different approach would be to find a qualitatively new type of evidence for imagepercept equivalence that is not susceptible to the three alternative explanations just discussed. The aim of this article is to present just such a new type of evidence. First the evidence will be reviewed, and then its implications for the relation between imagery and perception will be discussed. I will argue that this source of evidence has a special status in the debate over imagery and perception: It has the ability to be decisive in the face of the alternative accounts discussed earlier, where the conventional data of cognitive psychology data do not.

\section{Evidence From Neuropsychology}

A considerable number of findings from neuropsychology are relevant to the relation between imagery and perception, although even within neuropsychology there seems to be little awareness of the quantity and coherence of these many separate findings. The importance of this evidence is that it expands qualitatively, as well as quantitatively, the support for visual perceptual mechanisms in visual mental imagery. The relevant findings in neuropsychology can be roughly grouped into two categories: those that implicate the use of visual processing areas of the brain in visual imagery, and those that implicate shared functional mechanisms for visual imagery and visual perception, above and beyond the fact that they share common brain regions.

\section{Common Neural Substrates for Imagery and Perception}

Cortical visual processing begins in the accipital lobes, which contain primary and secondary visual cortex, and continues in

\footnotetext{
${ }^{1}$ Color effects in imagery would provide a clear case for visual, rather than nonvisual spatial representation, but interestingly no such effects have been found (see Pylyshyn, 1984). In fact, Finke and Schmidt's $(1977,1978)$ imagery-induced McCollough effect is obtained only for imaged lines on a real colored background and not for imaged color on a real black and white grating.
} 
the posterior parietal and temporal lobes, which contain modality-specific visual representations as well as multimodal representations. The earliest suggestion that imagery might involve the use of the visual areas of the brain came from case reports of cortically blind patients. Cortical blindness is loss of vision due to destruction of the occipital cortex. Many of these patients appear unable to use mental imagery, despite the relative preservation of other cognitive abilities (Brown, 1972; Symonds $\&$ Mackenzie, 1957). A systematic assessment of imagery ability in cortically blind patients with well-localized lesions could in principle provide strong evidence on the relation between the neural substates of visual imagery and visual perception; in practice, however, the documented cases do not provide sufficiently detailed information about the patients' impaired and intact cognitive abilities to be more than suggestive.

Stronger evidence that parts of the visual cortex participate in visual imagery comes from the use of regional cerebral blood flow and electrophysiological techniques for measuring and localizing brain activity in normal subjects. Regional cerebral blood flow provides a spatially precise method of measuring regional brain activity in normal humans, with increased blood flow indexing increased activity. Roland and Friberg (1985) examined regional cerebral blood flow while subjects rested and during three cognitive tasks: mental arithmetic (subtracting 3s starting at 50), memory scanning of an auditory stimulus (mentally jumping every second word in a well-known musical jingle), and visual imagery (visualizing a walk through one's neighborhood making alternating right and left turns starting at one's front door). Subjects were periodically queried as to their current answer (i.e., the number they were on in the mental arithmetic task, the word they were on the auditory rehearsal task, and the location they were at in the imagery task). This procedure yielded error rates, from which the authors concluded that the three tasks were equally difficult. In each of the 11 normal subjects tested, the pattern of blood flow in the visual imagery task showed massive activation of the posterior regions of the brain compared to the resting state, including the occipital lobe (the visual cortex proper) and posterior superior parietal and posterior inferior temporal areas important for higher visual processing. These are the same areas that normally show increased blood flow during visual-perceptual tasks (Mazziotta, Phelps, \& Halgren, 1983; Roland, 1982; Roland \& Skinhoj, 1981). Furthermore, these areas did not show increases in blood flow compared with the resting state in the other two cognitive tasks.

Roland and Friberg's (1985) results demonstrate visual cortical involvement in a fairly complex imagery task that includes both visualizing scenes from memory and transforming them (at each turn in the imaginary walk). Goldenberg, Podreka, Steiner, and Willmes (1987) devised a simpler imagery task, along with a control task differing from the imagery task only in the absence of imagery. Different groups of normal subjects were given the same auditorily presented lists of concrete words to learn under different instructional conditions: One group was told to just listen to the words and try to remember them, and the other group was told to visualize the referents of the words as a mnemonic strategy. Some subjects in the no-imagery group reported spontaneously imaging the words when questioned after the experiment, and they were reclassified as imagery condi- tion subjects. Recall was higher overall for the imagery group, as would be expected if these subjects did indeed differ from the no-imagery group in their use of imagery. The patterns of blood flow recorded during the two conditions also differed, by two distinct measures. First, there was relatively more blood flow to the occipital lobes, particularly the left inferior occipital region, in the imagery condition. Second, the pattern of covariation of blood flow among brain areas (calculated by a Smallest Space Analysis; Lingoes, 1979), which provides another index of regional brain activity, was also greater in the occipital and posterior temporal areas of the brain bilaterally in the imagery condition compared to the nonimagery condition.

Goldenberg, Podreka, Steiner, Deeke, \& Willmes (in press) compared the patterns of regional blood flow while subjects tried to answer several types of questions, among which were questions that require visual imagery to answer (e.g., "Is the green of pine trees darker than the green of grass?") and questions that do not require imagery to answer (e.g., "Is the categorical imperative an ancient grammatical form?"). Despite the superficial similarity of the two types of task, answering yes-no general knowledge questions, they differed significantly in the patterns of regional cerebral blood flow they evoked: The imagery questions caused greater occipital blood flow than did the nonimagery questions. The results of the Smallest Space Analy. sis also implicated occipital activity in the imagery condition, as well as revealing activity in the posterior temporal and posterior parietal visual processing areas. In contrast, the nonimagery condition did not reveal visual area activation.

Might the increased visual area activity in Goldenberg et al's (in press) imagery tasks merely index greater effort by subjects in those tasks than in the nonimagery control tasks? This is unlikely for three reasons. First, task effortfulness is generally reflected in blood flow changes to the frontal lobes, and has not been observed to produce occipital changes (Ingvar \& Risberg, 1967; Lassen, Ingvar, \& Skinhoj, 1978). Second, subjects in the first experiment who were given the more effortful task of memorizing lists of abstract words, rather than concrete words, under the same task conditions with no imagery instructions, did not show increased occipital blood flow. Third, whereas the imagery condition of Goldenberg et al.'s (1987) first experiment involved more effortful processing than the nonimagery condition, the imagery condition of Goldenberg et al.'s (in press) experiment was easier than the nonimagery condition (as evidenced by the lower error rates).

In three very different experimental paradigms, one a rather open-ended request to visualize a walk through familiar territory, another a verbal list-learning task in which imagery use was manipulated by explicit instructions, and the third a question-answering task in which imagery use was manipulated by implicit differences in the nature of the questions, convergent findings emerged: In each case, the imagery induced blood flow to the visual areas of the brain. Furthermore, in the two latter studies, the imagery conditions differed minimally from the comparison conditions, which did not show these increases.

Further evidence that the visual cortex participates in visual imagery comes from electrophysiological techniques: electroencephalography (EEG) and event-related potentials (ERP). In EEG techniques, suppression of alpha rhythm (EEG activity in a certain range of frequencies) is associated with increased 
brain activity. Many authors have found that visual imagery is accompanied by alpha rhythm attenuation over the visual areas of the brain (Barratt, 1956; Brown, 1966; Davidson \& Schwartz, 1977; Golla, Hutton, \& Gray Walter, 1943; Short, 1953; Slatter, 1960). Unfortunately, a methodological flaw in most of these studies is the lack of control for the degree of overall mental effort involved in the visual imagery and comparison conditions. However, the study of Davidson and Schwartz (1977) does contain the appropriate control measures and provides a clear and elegant demonstration of the modality-specific nature of the brain activity underlying imagery: Davidson and Schwartz measured the EEG alpha rhythm simultaneously over the visual (occipital) and tactile (parietal) areas of the brain, during visual imagery (imagining a flashing light), tactile imagery (imagining one's forearm being tapped), and combined visual and tactile imagery (imagining the flashes and taps together). Whereas there was no difference in total alpha attenuation between the visual and tactile imagery conditions (i.e., the overall effects of tactile and visual imagery on general effort and arousal were the same), the site of maximum alpha attenuation in the visual imagery condition was over the visual areas and the site of maximum alpha attenuation in the tactile imagery condition was over the tactile areas. Alpha attenuation in the combined visual and tactile imagery condition showed a more balanced pattern of distribution across both visual and tactile areas.

Recent work using ERP techniques offers another electrophysiological window on the areas of the brain engaged during imagery. Event-related potentials differ from EEG in that they measure only the electrical activity of the brain that is synchronized with (and thus presumably "related" to) the processing of a stimulus. Farah, Peronnet, Weisberg, and Perrin (1988) measured the ERP to visually presented words under two different instructional conditions: simply reading the words, and reading the words and imaging their referents (e.g., if the word is cat, imaging a cat). The words were presented for 200 ms each. The ERPs were recorded from 16 standard sites on the scalp, including occipital, parietal, temporal, and frontal locations. The first $450 \mathrm{~ms}$ of the ERPs in both conditions were indistinguishable, reflecting their common visual and lexical processing stages. However, later components of the two conditions differed from one another: In the imagery condition there was a highly localized increase in positivity of the ERP, relative to the reading-only condition, at the occipital electrodes, implicating occipital activity during the process of imaging. Scalp current density analyses of the ERP data, which provide enhanced localization (Perrin, Bertrand, \& Pernier, 1986) revealed a central occipital current source and lateral occipital current sinks, consistent with ERP generators in occipital cortex; and two occipito-temporal current sources and lateral fronto-temporal current sinks, consistent with an ERP generator in each temporal lobe.

Is it possible that this occipital ERP reflects general effects of cognitive load and is not specifically related to imagery? To test this possibility, subjects were presented with a new task, the misspelling detection task, which involved the same stimuli presented under the same conditions as the previous experiment. This experiment compared the reading-only of correctly spelled words to the detection of occasional misspellings, an effortful visual task using the same stimuli as the imagery task (except that about one in eight words was misspelled). The difference between the ERPs from reading and misspelling detection showed a different polarity as well as a different temporal and spatial distribution compared with the imagery effect observed earlier: This effect consisted of increased negativity rather than positivity, affecting a broader region of the posterior scalp (extending to the anterior temporal electrodes), and peaking about $200 \mathrm{~ms}$ earlier. Therefore, the focal occipital positivity observed when subjects form images is not merely a manifestation of a general "visual effort" effect on the evoked potential, but is tied more specifically to the processes taking place in the imagery condition of the experiment. Furthermore, when the imagery condition was changed in a subsequent experiment from one in which the subject images a different object from memory on each trial, to the repeated imaging of a small set of line drawings that subjects memorized just before ERP recording, the same focal occipital positivity ensued.

Farah, Peronnet, Gonon, and Giard (in press) took a different approach to localizing mental imagery in the brain using eventrelated potential techniques, by examining the effect of imagery on the ERP to visual stimuli. Subjects were instructed to image stimuli while they being presented with real stimuli, so that we could observe the effect of imagery on the ERP to stimuli. We reasoned that if imagery has a systematic effect on the ERP to stimuli, then there must be some common brain locus at which imagery and perceptual processing interact. More important, if the interaction between imagery and perception is content specific-that is, for example, if imaging an $H$ affects the ERP to $H$ s more than the ERP to $T s$, and imaging a $T$ affects the ERP to $T \mathrm{~s}$ more than the ERP to $\mathrm{Hs}$-then that interaction must be taking place at some locus where information about the differences between $H \mathrm{~s}$ and $T \mathrm{~s}$ is preserved, that is, at a representational locus. In this experiment, subjects imaged $H$ s and $T s$ while performing a detection task in which an $H$, a $T$, or no stimulus was presented on each trial. The image that the subject was instructed to form on a given trial was nonpredictive of the upcoming stimulus. The ERPs to $H$ s and Ts while subjects imaged the same letter were compared with the ERPs to $H$ s and Ts while subjects imaged the other letter. In this way, we could observe the content-specific effect of imagery on the visual ERP while holding constant the actual stimuli to which the ERPs were recorded (equal numbers of $H \mathrm{~s}$ and $T \mathrm{~s}$ in both conditions) and the effort of forming and holding an image (equal numbers of $H$ and $T$ images in each condition). If there is a content-specific effect of imagery on the visual ERP, then by localizing it we can put constraints on the location of representations accessed by both imagery and perception.

Imagery had a content-specific effect on the evoked potential within the first $200 \mathrm{~ms}$ of stimulus processing, and this effect was localized at the occipital recording sites. Furthermore, the inference that the underlying brain location of the image-percept interaction is occipital is strengthened by the fact that the time course of the effect of imagery on the ERP was the same as that of the first negative peak of the visual ERP waveform, which is believed to originate in occipital cortex (Lesevre \& Joseph, 1980; Maier, Dagnelie, Spekreijse, \& Van Dijk, 1987). The finding that an effect is maximal just when an ERP compo- 
nent is maximal implies that the neural locus of the effect is one or more of the generators of the ERP component.

To sum up the relevant electrophysiological literature, two measures, EEG and ERP, have been used in a variety of experiments involving imagery. In all cases, imagery activity was localized to the occipital regions. Furthermore, in a subset of this body of experiments (Davidson \& Schwartz, 1977; Farah, Peronnet, Weisberg \& Perrin, 1988; Farah, Peronnet, Gonon \& Giard, in press), control conditions were included which allow us to assess the cognitive specificity of these electrophysiological effects, and in each case they were associated with visual imagery activity per se. The electrophysiological evidence is thus in agreement with results from a very different methodology, regional cerebral blood flow, in implicating occipital activity during imagery. Across a variety of tasks, it has been found that imagery engages visual cortex, whereas other tasks, many of which are highly similar save for the absence of visual imagery, do not.

The most straightforward and parsimonious conclusion from this pattern of results is that mental images are visual representations, that is, they consist at least in part of some of the same representations used in vision. However, there does exist a logically correct alternative explanation according to which mental images are not visual representations, but are merely accompanied by activation in visual brain areas. On this account, the visual area activation is epiphenomenal with respect to the functions of imagery. To distinguish between these alternatives, we must find out whether destruction of visual brain areas results in imagery impairments as well as visual impairments. Parallel impairments in imagery and perception after brain damage imply that the visual areas implicated in the localization studies reviewed earlier do play a functional role in imagery, whereas the finding that imagery is unimpaired in patients with visual disorders following brain damage implies that activation of visual areas during imagery is epiphenomenal. The data reviewed in the next section allow us to distinguish between a functional and an epiphenomenal role for the visual system in imagery by reporting the effects of damage to the visual system on imagery ability. In addition, these data add quantitatively to the accumulating evidence for the involvement of the visual system in mental imagery.

\section{Functional Parallels Between Imagery and Perception After Brain Damage}

The existence of highly selective deficits in visual abilities has contributed to our understanding of the functional architecture of visual perception by demonstrating which perceptual abilities are independent of which other abilities. If visual imagery uses the same representational machinery as visual perception, then one should expect selective deficits in the imagery abilities of patients that parallel their selective perceptual deficits. In fact, for all of the types of selective visual deficits due to cortical lesions in which imagery has been examined, parallel imagery deficits have been observed.

At early stages of cortical visual processing, color is represented separately from other visual stimulus dimensions, and brain damage affecting the cortical visual areas can therefore result in relatively isolated color vision deficits (see Cowey,
1982; Meadows, 1974). A long history of the case-by-case study of patients with acquired cerebral color blindness has documented an association between loss of color perception and loss of color imagery (e.g., Beauvois \& Saillant, 1985, Case 2; Heidenhain, 1927; Jossman, 1929; Lewandowsky, 1908; Pick, 1908; Riddoch \& Humphreys, 1987; Sacks \& Wasserman, 1987; Stengel, 1948). In addition to being unable to identify or discriminate among colors, these patients cannot report the colors of common objects from memory (e.g., the color of a football, cactus or German Shepard's back), a task that most people find requires imaging the object in color. These patients are not generally impaired in their cognitive functioning. In fact, Sacks and Wasserman (1987) and Riddoch and Humphreys (1987) have documented good general imagery ability in their patients with acquired cerebral color blindness, as assessed by drawings and descriptions of objects from memory; the only aspect of imagery that was impaired was imagery for color. The implication of this association between the perception of color and imagery for color is that the two abilities depend upon the same neural substrates of color representation.

DeRenzi and Spinnler (1967) pointed out the need for a more systematic study of color-related impairments after brain damage, and undertook a large group study of unilaterally braindamaged patients in which they assessed color vision and color imagery. Color vision was tested in two ways: having the patient sort a set of colored paper squares into pairs having the same color, and having the patient name or trace out the digit embedded in random dots that are segregated into digit and background only by color (the "Ishihara" test of color blindness). Color recall was also tested in two ways: having the patient respond verbally to questions of the form "What color is a tangerine?" and "What color is cement?", and having the patients color black and white line drawings of objects with their characteristic color chosen from a set of colored crayons. DeRenzi and Spinnler found that patients who had impaired color vision also had impaired color imagery. Perhaps it is not surprising that a patient with a color vision deficit would perform poorly on the coloring task, in which color vision is needed to select the appropriate crayon, or that patients with language or memory impairments would do poorly on a verbal task of color memory. However, the relation between color vision impairment and color imagery impairment held high statistical significance even when patients who were neither language impaired nor memory impaired were considered on just the verbal test of color imagery.

Another source of evidence that color is represented by the same neural structures in imagery and perception comes from an intriguing case study by Beauvois and Saillant (1985, Case 1) of a patient whose visual areas had been neuroanatomically disconnected from her language areas by a stroke. The patient was able to perform color tasks that were purely visual, such as sorting objects on the basis of color and identifying the embedded characters in the Ishihara test of color blindness, because her visual areas had not been damaged. Her general verbal ability was also quite intact, as evidenced by a verbal IQ score of 123 , because her language areas had not been damaged. However, if the task involved coordinating a visual and verbal representation, for example, naming a visually presented color or pointing to a named color, her performance was extremely poor, 
owing to the neuroanatomical disconnection between her language and vision areas. The patient was tested on various color memory tasks, including two similar to those of DeRenzi and Spinnler (1967): viewing correctly and incorrectly colored drawings of objects and distinguishing between them, and answering verbally posed questions about the color of common objects of the form "What color is a - ?" The patient was able to perform the purely visual color memory task, implying that her mental images of colored objects were not disconnected from the visual areas used in recognizing and discriminating among the colored pictures. Her performance on the verbally posed color questions depended upon the nature of the question: For questions that made use of verbal associations between objects and colors (e.g., "What color is Paris ham?" where "Paris ham" is also called "white ham"; or "What color is envy?") the patient performed normally. In contrast, for questions that appear to require mental imagery (e.g., "What color is a gherkin?'), she performed poorly. Again, this implies that whereas verbal memory associations for colors were not disconnected from the language areas of this patient with visual-verbal disconnection, imagistic representations of color were. Finally, Beauvois and Saillant directly manipulated whether the patient used imagery or nonimagistic memory representations for retrieving the same information. In one condition, they asked questions such as "You have learnt what color snow is. It is often said. What do people say when they are asked what color snow is?" or "It is winter. Imagine a beautiful snowy landscape . . . Can you see it? Well, now tell me what color the snow is." The patient performed normally when biased toward a verbal recall strategy, and her performance dropped significantly when biased toward an imagery recall strategy. This is again what one would expect to find if the color of mental images is represented in the same neural substrate as the color of visual percepts.

In sum, three types of evidence support the hypothesis that imaging an object in color requires some of the same neural representations necessary for color vision: Individual cases of acquired central color blindness are reported to have lost their color imagery, in a group of patients with varying degrees of color vision impairment color imagery is correlated with color vision, and in a case of visual-verbal disconnection, images were equivalent to visual representations in terms of their interactions with other visual and verbal task components.

Patients with bilateral parieto-occipital disease often have trouble knowing where an object is in the visual field, without any difficulty identifying what the object is (DeRenzi, 1982). The impairment in the localization of stimuli in space may be quite selective to the visual modality, so that these patients can orient to tactile and auditory stimuli. At the same time, these patients are unimpaired in their ability to recognize of visual stimuli. Thus, such a patient may quickly identify an object such as a postage stamp held somewhere in his or her visual field, but may be unable to indicate its position either verbally or by pointing. Other patients, with bilateral temporo-occipital disease, may show the opposite pattern of visual abilities (Bauer \& Rubens, 1985). These "agnosic" patients are impaired in their ability to recognize visually presented stimuli, despite adequate elementary visual capabilities (e.g., size of visual field, acuity), and their failure of recognition is modality specific: They are able to recognize objects by touch or by characteristic sounds. Furthermore, their ability to localize visually presented objects is unimpaired. Thus, such a patient might fail to recognize a postage stamp by sight but could accurately point to its location. This dissociation is evidence for a rather counterintuitive division of labor in the visual system between the localization of stimuli and their identification, an idea which is also supported by animal experimentation (Ungerleider \& Mishkin, 1982). Levine, Warach, and Farah (1985) studied the imagery abilities of a pair of patients, one with visual localization impairment after bilateral parieto-occipital damage and one with visual object identification impairment after bilateral temporooccipital damage, with special attention to the distinction between spatial location information and single object appearance information in visual images. We found that the preserved and impaired aspects of vision in each patient were similarly preserved or impaired in imagery: The patient with object identification difficulties was unable to draw or describe the appearances of familiar objects, animals and faces from memory, despite being able to draw and describe in great detail the relative locations of cities and states on a map, furniture in his house, and landmarks in his city. The patient with object localization difficulties was unable to describe the relative locations of landmarks in his neighborhood, cities in the United States, or, when blindfolded, to point to furniture in his hospital room. He was, however, able to give detailed descriptions of the appearance of a variety of objects, animals, and faces. In a review of the literature for similar cases, we found that for a majority of the published cases of selective visual "what" or "where" deficit, when the appropriate imagery abilities were tested they showed parallel patterns of imagery deficit, and in no case was there a welldocumented violation of this parallelism: Of 28 cases of object identification difficulties in the literature, 14 were reported to have parallel imagery impairments, 6 were not examined regarding imagery, and 3 were reported to have intact imagery. For all 3 of this last group of patients, the authors of the case reports relied exclusively on the patients' own introspective assessments of their imagery ability. Of 26 cases of visual disorientation, imagery for spatial relations was tested in only 12 . In 9 of these cases it was found to be defective. Of the remaining 3 , the information concerning their imagery consisted of in one case having "good memory for paths in the city" with no other details given, in another case being able to "describe a geographic map" and in a third being able to describe the ward plan accurately. This third patient was unusual for a case of visual disorientation in that she was able to find her way about.

Dissociations between object recognition abilities within the temporo-occipital "what" system also exist. One form of dissociation that has been observed corresponds roughly to an impairment in recognizing living things (people, animals, and plants) with relatively better recognition of nonliving things. Farah, Hammond, Mehta, and Ratcliff (in press) studied a patient with this constellation of recognition abilities, comparing his ability to image the appearances of living and nonliving things. Imagery was tested by yes-no questions, such as "Are the hind legs of a kangaroo shorter than the front legs?" and "Does a guitar have a round-shaped hole in it?" The patient was significantly more impaired at recalling the appearances of living things than nonliving things, relative to the performance of age and education-matched normal subjects. His general knowl- 
edge about living and nonliving things was tested by similar yesno questions, such as "Is peacock served in French restaurants?" and "Were wheelbarrows invented before 1920?" In contrast to his ability to recall appearances, his ability to recall nonvisual information was normal for both living and nonliving things.

Beyn and Knyazeva (1962) compared the visual recognition and visual imagery abilities of an agnosic patient on an itemby-item basis for a small set of items. They found a close association between the particular visual stimuli that could be recognized and imaged: The patient recognized 3 out of 16 items that he was unable to image (as assessed by drawings from memory) and 13 out of 16 objects that he could image.

The most selective deficit of visual object recognition consists of profoundly impaired face recognition with roughly intact recognition of other classes of visual stimuli as well as intact general intellectual and memory functioning. Shuttleworth, Syring, and Allen (1982) examined the relation between face recognition and face imagery in a patient with a selective face recognition deficit (their Case 2) and in the published literature on face recognition deficits. Their patient was reported to have "no voluntary visual recall (revisualization) of faces but was able to revisualize more general items such as buildings and places" (p. 313). Shuttleworth et al. found that approximately $40 \%$ of 74 cases of face recognition deficit in the neurology literature reported impairments in face imagery. They went on to caution that in many of the cases in which face imagery was not noted to be impaired, "the accuracy of the image could not be ascertained and was seriously questioned in a number of cases" (p. 313).

Patients with right-parietal-lobe damage often fail to detect stimuli presented in the left half of the visual field, even though their elementary sensory processes for stimuli on the affected side of space are intact (Heilman, Watson, \& Valenstein, 1985; Posner, Walker, Friedrich, \& Rafal, 1984). This deficit is known as "visual neglect," and also appears to manifest itself in visual imagery. Bisiach and his colleagues (Bisiach \& Luzzatti, 1978; Bisiach, Luzzatti, \& Perani, 1979) have shown that right-parietal patients with visual neglect also fail to access the left sides of imagined objects and scenes. In Bisiach and Luzzatti's initial report, two right-parietal-lobe-damaged neglect patients were asked to imagine viewing a famous square in Milan (the Piazza del Duomo, with which the patients had been familiar before their brain damage) from a particular vantage point, and to describe the view. Both patients omitted from their descriptions the landmarks that would have fallen on the left side of that scene. The patients were then asked to repeat the task, this time from the opposite vantage point, from which the buildings, statues, and other landmarks that fell on the left side of the previous view were visible on the right, and vice versa. The patients' descriptions of their images now included the items that had previously been omitted, and omitted the items on the left side of their current image (which had before been reported).

Bisiach et al. (1979) followed up these case studies with a group study of neglect for visual images. Right-parietal-lobedamaged patients with left-sided neglect and a control group of patients without neglect were shown abstract cloud-like shapes passing behind a screen with a narrow vertical slit in the center. Because all of the stimulus input in this task is presented cen- trally in the visual field, any effect of left-sided neglect in this task cannot be attributed to perceptual neglect. After viewing pairs of such shapes, the patients were to decide whether the two members of the pair were identical or different. This presumably requires mentally reconstructing images of the stimuli from the successive narrow vertical views. Patients who neglected the left halves of visual stimuli also neglected the left halves of their images, as evidenced by a greater number of errors when pairs of shapes differed on their left sides than when they differed on their right sides in the task.

\section{Discussion}

It was shown earlier that the evidence for visual mechanisms in imagery from cognitive psychology is susceptible to three specific lines of criticism. Can the same be said of the neuropsychological evidence summarized earlier? To examine this, let us review each of the alternative explanations and attempt to apply them to the present data. A tacit knowledge account of the electrophysiological and blood flow data, implicating the use of cortical visual areas during visual imagery activity, would need to include the following two assumptions: (a) that subjects know what parts of their brains are normally active during vision and (b) that subjects can voluntarily alter their brain electrical activity, or modulate or increase regional blood flow to specific areas of their brains. It is clear that most subjects do not consciously know which brain areas are involved in vision, but what about the possibility of tacit knowledge? Tacit knowledge of the neural localization of visual processing would be impossible to acquire: Whereas one could conceive of mechanisms by which a subject might acquire tacit knowledge of many subtle functional properties of his or her visual system (by observing aftereffects, illusions, the relative difficulty of seeing different stimuli, etc.), there are no conceivable mechanisms by which a subject could gain tacit knowledge of the neuroanatomical locations of visual processing. The second assumption is also difficult to accept; whereas subjects can learn through biofeedback techniques to modulate EEG spectra, for example, untrained subjects cannot voluntarily change features of their EEG (Nowlis \& Kamiya, 1970).

How would the tacit knowledge account explain functional parallels observed between perceptual and imaginal deficits after brain damage? As with normal subjects, the assumption would be made that the patients take their task to be behaving as if they were actually seeing the to-be-imagined stimuli. But this answer does not entirely constrain a prediction, because we do not know whether patients who know they have visual deficits would behave as if they were seeing with normal visual systems (i.e., using their tacit knowledge of normal vision) or with their defective visual systems (i.e., using their more recently acquired tacit knowledge of their impaired vision). An independent basis for deciding between these two predictions comes from studies of subjects who were peripherally (as opposed to cortically) blinded late in life. These subjects perform essentially normally on visual imagery tasks (Hollins, 1985). In terms of a tacit knowledge account of performance in imagery tasks, this implies that patients with visual deficits will interpret imagery tasks as demanding the simulation of intact visual processes. This leads to the prediction that patients with acquired visual 
disorders of cerebral origin should continue to perform normally in imagery experiments, a prediction which is clearly disproved by the available evidence.

Even if it is assumed that, unlike the patients with peripheral visual disorders, the patients with central visual disorders make the strategic decision to tailor their imagery task performance to match their own, defective, perceptual performance, several problems remain for the tacit knowledge account. First, whereas normal subjects in imagery tasks would be modulating subtle properties of their responses (such as response latency) to simulate visual processes, patients would be feigning an inability to perform certain imagery tasks. It is somewhat implausible that patients would persist in failing easy tasks when they could be giving correct responses. Second, studies of malingering patients, who do intentionally perform poorly on neuropsychological tests, have shown that statistical naivete leads them to perform significantly worse than chance (Lezak, 1983), which is not the case with the patients in the studies reviewed earlier. A final difficulty with the tacit knowledge account is specific to the findings on visual neglect in imagery: Most patients with visual neglect deny that they have any visual difficulty, and their behavior of leaving uneaten food on the left sides of their plates when they are hungry, injuring themselves by walking into objects on their left sides, and so on evinces a lack of even tacit knowledge of this deficit (Heilman et al., 1985). The two patients in Bisiach and Luzzatti's (1978) case studies were both unaware of their visual difficulty, and one may assume that if their group study included typical patients, then these subjects too would have been unaware of their deficits. Nevertheless, and contrary to the tacit knowledge hypothesis, these patients demonstrated parallel deficits in their imagery performance.

Could experimenter expectancy have produced some or all of the neuropsychological evidence reviewed here? In the case of the observed parallels between perceptual and imaginal deficits this possibility certainly exists, but it is less likely than in the corresponding cognitive literature because of the wide range of investigators, whose work spans several decades before the current "imagery debate," and the majority of whom had no stated position on the issue of the relation between imagery and perception. Nonetheless, the effects of experimenter expectancy on this data cannot strictly be ruled out. In contrast, the EEG, ERP, and blood flow findings represent psychophysiological measures that would be impossible to "shape" by the normal mechanisms of experimenter expectancy in psychological research. Unless the two assumptions needed for a tacit knowledge account of these findings are granted, namely, that subjects know where their visual processing areas are and have the ability to tailor their EEG, ERP, and blood flow accordingly, there is no way that instructions given prior to the recording of EEG, ERP, or blood flow could produce the results actually obtained in these studies. For most of the studies, communication from the experimenters during the recording sessions could not affect the results through a biofeedback mechanism either: In most of the EEG studies (including Davidson \& Schwartz, 1977), and in both of the ERP studies, subjects were isolated from the experimenters during data collection.

How do the neuropsychological results fit in with the observations that peripherally blind subjects can use imagery? Far from being at odds with one another, these two sets of findings to- gether make clear the sense in which visual imagery is visual. Specifically, imagery is not visual in the sense of necessarily representing information acquired through visual sensory channels. Rather, it is visual in the sense of using some of the same neural representational machinery as vision. That representational machinery places certain constraints on what can be represented in images and on the relative ease of accessing different kinds of information in images. It is possible that peripherally blind subjects, even those blind from birth, can use their intact cortical visual areas for internal representation during imagery tasks. It is also possible to explain the performance of congenitally blind subjects in imagery tasks in terms of nonvisual spatial representations, as Kerr and others have proposed, without being forced to suppose that normal subjects perform these tasks the same way. Given that the brain represents spatial information with auditory, tactile, and visual modality-specific representations, it is not unparsimonious to assume that normal subjects have a choice of using visual or nonvisual spatial representations for performing imagery tasks (cf. Davidson \& Schwartz, 1977), and that the extent of a subject's visual experience or deprivation would determine which of these representations is chosen.

\section{References}

Anderson, J. R. (1983). The architecture of cognition. Cambridge, MA: Harvard University Press.

Atwood, G. E. (1971). An experimental study of visual imagination and memory. Cognitive Psychology, 2, 290-299.

Baddeley, A. D., \& Lieberman, K. (1980). Spatial working memory. In Attention and performance VIII (pp. 521-539). Hillsdale, NJ: Erlbaum.

Barratt, P. E. (1956). Use of the EEG in the study of imagery. British Journal of Psychology, 47, 101-114.

Bauer, R. M., \& Rubens, A. B. (1985). Agnosia. In K. M. Heilman \& E. Valenstein (Ed.), Clinical neuropsychology (2nd ed.)(pp. 187-241). New York: Oxford University Press.

Beauvois, M. F., \& Saillant, B. (1985). Optic aphasia for colours and colour agnosia: A distinction between visual and visuo-verbal impairments in the processing of colours. Cognitive Neuropsychology, 2(1), $1-48$.

Beyn, E. S., \& Knyazeva, G. R. (1962). The problem of prosopagnosia. Journal of Neurology, Neurosurgery and Psychiatry. 25, 154-158.

Bisiach, E., \& Luzzatti, C. (1978). Unilateral neglect of representational space. Cortex, 14, 129-133.

Bisiach, E., Luzzatti, C., \& Perani, D. (1979). Unilateral neglect, representational schema and consciousness. Brain, 102, 609-618.

Block, N. (1983). Mental pictures and cognitive science. The Philosophical Review, 92, 499-541.

Broerse, J., \& Crassini, B. (1980). The influence of imagery ability on color aftereffects produced by physically present and imagined induction stimuli. Perception \& Psychophysics, 28, 560-568.

Broerse, J., \& Crassini, B. (1981). Misinterpretations of imagery-induced McCollough effects: A reply to Finke. Perception \& Psychophysics, 30, 96-98.

Broerse, J., \& Crassini, B. (1984). Investigations of perception and imagery using CAEs: The role of experimental design and psychophysical method. Perception \& Psychophysics, 35, 153-164.

Brown, B. B. (1966). Specificity of EEG phoptic flicker responses to color as related to visual imagery ability. Psychophysiology, 2(3), 197 207.

Brown, J. W. (1972). Aphasia. apraxia and agnosia: Clinical and theoretical aspects. Springfield, IL: Charles $\mathrm{C}$. Thomas. 
Carpenter, P. A., \& Eisenberg, P. (1978). Mental rotation and the frame of reference in blind and sighted individuals. Perception \& Psychophysics, 23, 117-124.

Cowey, A. (1982). Sensory and non-sensory visual disorders in man and monkey. In D. E. Broadbent \& L. Weiskrantz (Eds.), The neuropsychology of cognitive function (pp. 3-13). London: The Royal Society,

Davidson, R. J., \& Schwartz, G. E. (1977). Brain mechanisms subserving self-generated imagery: Electrophysiological specificity and patterning. Psychophysiology; 14, 598-601.

DeRenzi, E. (1982). Disorders of space exploration and cognition. New York: John Wiley \& Sons.

DeRenzi, E., \& Spinnler, H. (1967). Impaired performance on color tasks in patients with hemispheric lesions. Cortex, 3, 194-217.

Farah, M. J., Hammond, K. H., Mehta, Z., \& Ratcliff, G. (in press). Category specificity and modality specificity in semantic memory. Neuropsychologia.

Farah, M. J., Peronnet, F., Gonon, M. A., \& Giard, M. H. (in press). Electrophysiological evidence for a shared representational medium for visual images and percepts. Journal of Experimental Psychology: General.

Farah, M. J., Peronnet, F., Weisberg, L. L., \& Perrin, F. (1988). Brain activity underlying mental imagery: An ERP study. Manuscript submitted for publication.

Finke, R. A. (1979). The functional equivalence of mental images and errors of movement. Cognitive Psychology, 11, 235-264.

Finke, R. A. (1980). Levels of equivalence in imagery and perception. Psychological Review, 87, 113-132.

Finke, R. A. (1985). Theories relating mental imagery to perception. Psychological Bulletin, 98, 236-259.

Finke, R. A., \& Kosslyn, S. M. (1980). Mental imagery acuity in the peripheral visual field. Journal of Experimental Psychology: Human Perception and Performance, 6, 244-264.

Finke, R. A., \& Kurtzman, H. S. (1981). Mapping the visual field in mental imagery. Journal of Experimental Psychology: General, 110, 501-517.

Finke, R. A., \& Schmidt, M. J. (1977). Orientation-specific color aftereffects following imagination. Journal of Experimental Psychology: Human Perception and Performance, 3, 599-606.

Finke, R. A., \& Schmidt, M. J. (1978). The quantitative measure of pattern representation in images using orientation-specific color aftereffects. Perception \& Psychophysics, 23, 515-520.

Fodor, J. A. (1983). The modularity of mind. Cambridge, MA: MIT Press.

Goldenberg, G., Podreka, I., Steiner, M., Suess, E., Deeke, L., \& Willmes, K. (in press). Regional cerebral blood flow patterns in imagery tasks-results of single photon emission computer tomography. In M. Denis, J. Engelkamp, \& J. T. E.Richardson (Eds.), Cognitive and neuropsychological approaches to mental imagery. Dodrecht, The Netherlands: Martinus Nijhoff.

Goldenberg, G., Podreka, I., Steiner, M., \& Willmes, K. (1987). Patterns of regional cerebral blood flow related to memorizing of high and low imagery words: An emission computer tomography study. Neuropsychologia, 25, 473-486.

Golla, F. L., Hutton, E. L., \& Gray Walter, W. G. (1943). The objective study of mental imagery. I. Physiological concomitants. Journal of Mental Science, 75, 216-223.

Hebb, D. O. (1968). Concerning imagery. Psychological Review, 75 , 466-479.

Heidenhain, A. (1927). Beitrag zur kenntnis der seelenblindheit [contribution to the knowledge of mindblindness]. Monatsschrift fur Psychiatric und Neurologie, 65, 61-116.

Heilman, K. M., Watson, R. T., \& Valenstein, E. (1985). Neglect and related disorders. In K. M. Heilman \& E. Valenstein (Eds.), Clinical neuropsychology (2nd ed.)(pp. 243-293). New York: Oxford University Press.

Hollins, M. (1985). Styles of mental imagery in blind adults. Neuropsychologia, 23, 561-566.

Hume, D. (1969). A treatise in human nature. Baltimore: Pelican Books. (Original work published 1739)

Ingvar, D. H., \& Risberg, J. (1967). Increase of regional cerebral blood flow during mental effort in normals and in patients with local brain disorders. Experimental Brain Research, 3, 195-211.

Intons-Peterson, M. J. (1983). Imagery paradigms: How vulnerable are they to experimenters' expectations? Journal of Experimental Psychology: Human Perception and Performance, 9, 394-412.

Intons-Peterson, M. J., \& White, A. R. (1981). Experimental naivete and imaginal judgments. Journal of Experimental Psychology: Human Perception and Performance, 7, 833-843.

Jossman, P. (1929). Zur psychopathologie des optisch-agnostichen storungen [on the psychopathology of optical agnosic disturbances]. Monatsschrift fur Psychiatric und Neurologie, 72, 81-149.

Kerr, N. H. (1983). The role of vision in visual imagery experiments: Evidence from the congenitally blind. Journal of Experimental Psychology: General, 112, 265-277.

Kosslyn, S. M. (1975). Information representation in visual images. Cognitive Psychology, 7, 341-370.

Kosslyn, S. M. (1980). Image and mind. Cambridge, MA: Harvard University Press.

Kosslyn, S. M. (1983). Ghosts in the mind's machine. New York: Norton.

Lassen, N. A., Ingvar, D. H., \& Skinhoj, E. (1978). Brain function and blood flow. Scientific American, 239, 62-71.

Lesevre, N., \& Joseph, J. P. (1980). Hypotheses concerning the most probable origins of the various components of the pattern evoked potential. In C. Barber (Ed.), Evoked potentials (pp. 159-166). MTP Press.

Levine, D. N., Warach, J., \& Farah, M. J. (1985). Two visual systems in mental imagery: Dissociation of 'what' and 'where' in imagery disorders due to bilateral posterior cerebral lesions. Neurology, 35, 10101018.

Lewandowsky, M. (1908). Ueber abspaltung des farbensinnes [about divisions of color sense]. Monatsschrif fur Psychiatric and Neurologie. 23, 488-510.

Lezak, M. (1983). Neuropsychological assessment (2nd ed.). New York: Oxford University Press.

Lingoes, J. C. (1979). The Guttman-Lingoes nonmetric program series. Ann Arbor, MI: Mathesis Press.

Maier, J., Dagnelie, G., Spekreijse, H., \& Van Dijk, B. W. (1987). Principle components analysis for source localization of visual evoked potentials in man. Vision Research, 12, 165-177.

Marmor, G. S., \& Zabeck, L. A. (1976). Mental rotation by the blind: Does mental rotation depend on visual imagery? Journal of Experimental Psychology: Human Perception and Performance, 2, 515-521.

Mazziotta, J. C., Phelps, M. E., \& Halgren, E. (1983). Local cerebral glucose metabolic response to audiovisual stimulation and deprivation: studies in human subjects with positron CT. Human Neurobiology, 2, 11-23.

Meadows, J. C. (1974). The anatomical basis of prosopagnosia. Journal of Neurology, Neurosurgery, and Psychiatry, 37, 489-501.

Neisser, U., \& Kerr, N. (1973). Spatial and mnemonic properties of visual images. Cognitive Psychology, 5, 138-150.

Nowlis, D. P., \& Kamiya, J. (1970). The control of electroencephalographic alpha rhythms through auditory feedback and associated mental activity. Journal of Neuroscience, 6, 476-484.

Perrin, F., Bertrand, O., \& Pernier, J. (1986). Scalp current density mapping: Value estimation from potential data. IEEE: Biomedical Engineering, 34, 283-288. 
Pick, A. (1908). Arbeiten aus den deutschen psychiatrischen Universitaetsklinik in Prag [Reports from the German Psychiatric Clinic in Prague]. Berlin: Krager.

Podgorny, P., \& Shepard, R. N. (1978). Functional representations common to visual perception and imagination. Journal of Experimental psychology: Human Perception and Performance, 9, 21-35.

Posner, M. I., Walker, J. A., Friedrich, F. J., \& Rafal, R. D. (1984). Effects of parietal lobe injury on covert orienting of visual attention. Journal of Neuroscience, 4, 1863-1874.

Pylyshyn, Z. W. (1973). What the mind's eye tells the mind's brain: A critique of mental imagery. Psychological Bulletin, 80, 1-24.

Pylyshyn, Z. W. (1978). Imagery and artificial intelligence. In C. W. Savage (Ed.), Perception and cognition: Minnesota studies in the philosophy of science (pp. 19-55). Minneapolis: University of Minnesota Press,

Pylyshyn, Z. W. (1981). The imagery debate: Analogue media versus tacit knowledge. Psychological Review, 88, 16-45.

Pylyshyn, Z. W. (1984). Computation and cognition. Cambridge, MA: MIT Press.

Riddoch, M. J., \& Humphreys, G. W. (1987). A case of integrative visual agnosia. Brain, 110, 1431-1462

Roland, P. E. (1982). Cortical regulation of selective attention in man. Journal of Neurophysiology, 48, 1059-1078.

Roland, P. E., \& Friberg, L. (1985). Localization of cortical areas activated by thinking. Journal of Neurophysiology, 53, 1219-1243.

Roland, P. E., \& Skinhoj, E. (1981). Focal activation of the cerebral cortex during visual discrimination in man. Brain Research, 222, 166-171.
Sacks, O., \& Wasserman, R. (1987, November 19). The case of the colorblind painter. New York Review of Books, 25-34.

Shepard, R. N. (1978). The mental image. American Psychologist, 33 125-137.

Shepard, R. N. (1984). Kinematics of perceiving, imagining, thinking, and dreaming. Psychological Review, 91, 417-447.

Shepard, R. N., \& Cooper, L. A. (1982). Mental images and their transformations. Cambridge, MA: MIT Press.

Short, P. L. (1953). The objective study of mental imagery. British Journal of Psychology, 44, 38-51.

Shuttleworth, E. C., Syring, V., \& Allen, N. (1982). Further observations on the nature of prosopagnosia. Brain and Cognition, 1, 302-332.

Slatter, K. H. (1960). Alpha rhythm and mental imagery. Electroencephalography and Clinical Neurophysiology, 12, 851-859.

Stengel, E. (1948). The syndrome of visual alexia with colour agnosia. Journal of Mental Science, 94, 46-58.

Symonds. C., \& Mackenzie, I. (1957). Bilateral loss of vision from cerebral infarction. Brain, 80, 28-448.

Ungerleider, L. G., \& Mishkin, M. (1982). Two cortical visual systems. In D. J. Ingle, M. A. Goodale, \& R. J. W. Mansfield (Eds.), Analysis of visual behavior (pp. 549-586). Cambridge, MA: MIT Press,

Zimler, J., \& Keenan, J. M. (1983). Imagery in the congenitally blind: How visual are visual images? Journal of Experimental Psychology: Learning, Memory, and Cognition, 9, 269-282.

Received April 30, 1986

Revision received September 17, 1987 Accepted September 30, 1987

\section{APA Buys Clinician's Research Digest}

APA has acquired the Clinician's Research Digest and will take over formal publication of the digest as of July 1, 1988. Presently published by the California-based Relational Dynamics Institute. $C R D$ offers practitioners brief summaries of clinically relevant research findings and other clinical information.

Clinton W. McLemore, PhD, president of Relational Dynamics, founded CRD in 1983. McLemore will continue to serve as $C R D$ editor through June 30, 1988. A new editor, to be selected, will take over as of July $1,1988$.

The CRD acquisition was proposed by the ad hoc Committee on Practitioner Publications (PPC), chaired by Charles D. Spielberger. From 1984 to 1987, the PPC, established by the P\&C Board at the behest of the BOD's Subcommittee on the Future of Professional Education in Psychology, made several recommendations for practice-oriented publications tailored for health service providers, school/educational psychologists, and I/O psychologists-including the development of monograph series for each group.

A continuing education program, which has also been acquired by APA, is offered in conjunction with the $C R D$. RDI will continue to operate the program under license from APA.

For the present, information on subscriptions to CRD and the CRD CE Program can be obtained from Clinical Information Services, P.O. Box 61025, Pasadena, California 911069990. $C R D$ will be issued monthly beginning in January 1988.1988 subscription rates: individuals, $\$ 48$; institutions, $\$ 62$. 